\begin{tabular}{l|l|l|l|l|l}
\hline Intropica & ISSN 1794-161X & Vol. 13 & No. 1 & $11-12$ & Santa Marta-Colombia, enero-junio de 2018 \\
\hline
\end{tabular}

\title{
RETOS DEL MILENIO: LA POBREZA EXTREMA Y EL HAMBRE, UNA REFLEXIÓN
}

\author{
CHALLENGES OF THE NEW MILLENIUM: A REFLECTION ON EXTREME \\ POVERTY AND HUNGER
}

Pedro Eslava-Eljaiek (1)

A medida que la población mundial crece, los desafíos para suplir las necesidades básicas de una población de más de 7500 millones de habitantes cobran importancia, sobre todo en aspectos que fueron formulados como retos del milenio por las Naciones Unidas (ONU, 2000) como la disminución de la pobreza extrema y del hambre. A pesar de que el informe de ese mismo organismo señala que en el 2015 esos indicadores se redujeron a más de la mitad, puesto que cayeron de 1900 millones en 1990 a 836 millones en 2015 (ONU, 2015), el desafío aún persiste, ya que las proyecciones de la ONU señalan que en 2020 la población mundial alcanzará los 7,7 mil millones y en 2100 la Tierra tendrá 11,2 mil millones de habitantes; los países en desarrollo fueron los de mayor crecimiento (UNFPA, 2011). Lo anterior indica que los requerimientos proteínicos y otros nutrientes esenciales para el sustento de esa población creciente deben derivarse de fuentes diversas a las del sector agropecuario.

Las proyecciones muestran que para alimentar una población mundial de 9100 millones de personas en 2050 sería necesario aumentar la producción de alimentos en un $70 \%$ entre los años 2005 y 2050 (FAO, 2009), por lo cual la producción de alimentos en los países en desarrollo tendría que duplicarse. Ello implicará un aumento importante en la producción de varios productos básicos fundamentales.

Así mismo, se tiene previsto que el $90 \%$ del crecimiento en la producción agrícola a nivel mundial $(80 \%$ en los países en desarrollo) se deberá a rendimientos más altos y a la intensificación de cultivos, y el resto a la ampliación de la superficie de las tierras. La superficie de tierras cultivables se incrementaría en unos 70 millones de hectáreas (menos del $5 \%$ ); este aumento quedaría contrarrestado en los países en desarrollo, donde sería de alrededor de 120 millones de hectáreas (12 \%) por una reducción de unos 50 millones de hectáreas (8 \%). La mayor parte de este aumento de superficie en los países en desarrollo tendría lugar en el África subsahariana y América Latina (FAO, 2009).

Sectores tan importantes como la pesca y la acuicultura tendrán un papel muy determinante en ese reto. Según el informe SOFIA de la FAO (2016), la producción mundial de pesca y acuicultura alcanzó en el año 2014 la suma de 167,2 millones de toneladas. La acuicultura fue el sector que porcentualmente tuvo el mayor crecimiento al pasar de producir 70,3 millones de toneladas en el año 2013 a producir 73,8 millones de toneladas en el 2014; es decir, un crecimiento del $4,97 \%$ frente al crecimiento porcentual de las capturas que fue del $0,75 \%$.

La investigación en la apuesta para buscar nuevas fuentes de energía en los niveles bajos de la cadena alimenticia y fortalecer aún más el desarrollo acuícola juega un importante protagonismo, dado que es un punto de partida para que las comunidades de peces, crustáceos y moluscos, tanto en el medio natural como en los cultivos bajo condiciones controladas, puedan convertirse en alternativas seguras para suplir esas demandas. En este sentido, la investigación a nivel marino en especies como los moluscos bivalvos ha demostrado ser una gran alternativa productiva en Colombia (Velasco, 2013).

Actualmente, investigadores asociados a la Universidad del Magdalena, otras instituciones de educación superior y centros de investigación del país vienen indagando en temas relacionados con redes tróficas en

\section{Dirección de los autores:}

Universidad del Magdalena, Instituto de Investigaciones Tropicales. Grupo de Investigación "Biodiversidad y Ecología Aplicada”. Carrera 32 No 22-08. Santa Marta, pedroeslavaeljaiek@gmail.com (P.E.E). 
ambientes acuáticos y cuyos logros podrán contribuir al fortalecimiento de la acuicultura y hacer aportes significativos en el incremento de la producción de organismos acuáticos bajo condiciones controladas con lo cual se podría hacer una contribución importante desde nuestro país a la disminución de la pobreza extrema y del hambre. Algunas de esas investigaciones se relacionan con la identificación de macroinvertebrados acuáticos asociados a las macrófitas acuáticas en quebradas de zonas insulares como el archipiélago de San Andrés (Cortés-Guzmán y Ospina-Torres, 2014) o el lago de Tota (Pedroza-Ramos y Caraballo, 2016) las cuales hacen parte de la dieta de peces como la trucha. También se destacan investigaciones en Eichhornia crassipes (Rúa-García, 2015), la cual abunda en los ecosistemas lénticos de muchos países tropicales y que es sustrato para muchas especies de invertebrados acuáticos. Así mismo, el estudio del ficoperitifton asociado a esas mismas macrófitas (Mogollón al, 2014; Osorio-Ávila y Manjarrés-García, 2015; Martínez-Silva, 2015) son de significativa importancia en la búsqueda de alternativas en la alimentación de organismos acuáticos y en la transferencia de energía en la cadena trófica.

Somos conscientes de que la Universidad del Magdalena, a través de sus grupos de investigación, seguirá buscando respuestas mediante sus iniciativas científicas en los diferentes ámbitos del conocimiento, no solamente para contribuir a disminuir la pobreza extrema y el hambre, sino para contribuir en el logro de objetivos del milenio como: alcanzar la enseñanza universal, promover la igualdad de género, reducir la mortalidad infantil, mejorar la salud materna, combatir el VIH/SIDA, el paludismo y otras enfermedades, garantizar la sostenibilidad del medio ambiente y fomentar la asociación mundial para el desarrollo, y de esta manera contribuir en la mejora en el nivel de vida de la población en nuestro país.

\section{REFERENCIAS}

Cortés-Guzmán, D. y Ospina-Torres, R. 2014. Comunidad de macroinvertebrados acuáticos en quebradas de la Isla de Providencia, mar Caribe colombiano. Intropica 9: 9-22.
División de Información y Relaciones Externas del UNFPA, Fondo de Población de las Naciones Unidas. Estado de la población mundial 2011. https://www.unfpa.org/sites/ default/files/pub-pdf/SP-SWOP2011_Final.pdf. Consultado: 22 de febrero 2018.

FAO. 2009. Cómo alimentar al mundo 2050. Foro de expertos de alto nivel. URL_http://www.fao.org/fileadmin/ templates/wsfs/docs/synthesis_papers/C \% C3\%B3mo_ alimentar_al_mundo_en_2050.pdf. Consultado: 31 de enero de 2018.

FAO. 2016. El estado mundial de la pesca y la acuicultura 2016. Contribución a la seguridad alimentaria y la nutrición para todos. Organizaciones para las Naciones Unidas para la alimentación y la Agricultura, Roma.

Martínez-Silva, P. 2015. Variación espacio-temporal de microalgas acuáticas del embalse de Betania-Huila y su relación con la calidad del agua. Intropica 10: 11-19.

Mogollón, M., Aycardi, M. P., Galeano, J., Villalobos, J. y Arango, C. 2014. Variación espacio-temporal de las cianoprocariotas del antiguo delta del río Sinú, Córdoba, Colombia. Intropica 9: 92-101.

Organización de las Naciones Unidas (ONU). 2000. Declaración del Milenio. Octava sesión plenaria 8 de septiembre de 2000, Nueva York.

Organización de las Naciones Unidas (ONU). 2015. Objetivos de Desarrollo del Milenio Informe de 2015. http://www.un.org/es/millenniumgoals/pdf/2015/mdgreport-2015_spanish.pdf. Consultado: 30 de enero de 2018.

Osorio-Ávila, F. y Manjarrés-García, G. 2015. Ficoperifiton asociado a macrófitas en la ciénaga Cerro de San Antonio, Magdalena-Colombia. Intropica 10: 74-83.

Pedroza-Ramos, A., Caraballo, P. y Aranguren-Riaño, N. 2016. Estructura trófica de los invertebrados acuáticos asociados a Egeria densa (Planch. 1849) en el lago de Tota, (Boyacá-Colombia). Intropica 11: 21-34.

Rúa-García, G. 2015. Macroinvertebrados acuáticos asociados a raíces de Eichhornia crassipes (MART) Solms en la ciénaga de Zapayán, Colombia. Intropica 10: 52-59.

Velasco, L.A. 2013. Esfuerzo reproductivo en moluscos: una revisión. Intropica 8: 87-97. 